\title{
CONVEX ISOMETRIC FOLDING
}

\author{
E. M. ELKHOLY
}

(Received 27 September 1993 and in revised form 5 June 1996)

\begin{abstract}
We introduce a new type of isometric folding called "convex isometric folding." We prove that the infimum of the ratio $\operatorname{Vol} N / \operatorname{Vol} \varphi(N)$ over all convex isometric foldings $\varphi: N \rightarrow N$, where $N$ is a compact 2-manifold (orientable or not), is $1 / 4$.
\end{abstract}

Keywords and phrases. Isometric and convex foldings, regular and universal covering spaces, manifolds, group of covering transformations, fundamental regions.

2000 Mathematics Subject Classification. Primary 51H10, 57N10.

1. Introduction. A map $\varphi: M \rightarrow N$, where $M$ and $N$ are $C^{\infty}$ Riemannian manifolds of dimensions $m$ and $n$, respectively, is said to be an isometric folding of $M$ into $N$ if and only if for any piecewise geodesic path $\gamma: J \rightarrow M$, the induced path $\varphi \circ \gamma: J \rightarrow N$ is a piecewise geodesic and of the same length. The definition is given by Robertson [4]. The set of all isometric foldings $\varphi: M \rightarrow N$ is denoted by $\mathscr{g}(M, N)$.

Let $p: M \rightarrow N$ be a regular locally isometric covering and let $G$ be the group of covering transformations of $p$. An isometric folding $\phi \in \mathscr{g}(M)$ is said to be $p$-invariant if and only if for all $g \in G$ and all $x \in X, p(\varphi(x))=p(\varphi(g, x))$. See Robertson and Elkholy [5]. The set of $p$-invariant isometric foldings is denoted by $\mathscr{F}_{i}(M, p)$.

DEFINITION 1.1. Let $\varphi \in \mathscr{g}(M, N)$, where $M$ and $N$ are $C^{\infty}$ Riemannian manifolds of dimensions $m$ and $n$, respectively. We say that $\varphi$ is a convex isometric folding if and only if $\varphi(M)$ can be embedded as a convex set in $\mathbb{R}^{n}$.

We denote the set of all convex isometric foldings of $M$ into $N$ by $C(M, N)$, and if $C(M, N) \neq \emptyset$, then it forms a subsemigroup of $\mathscr{g}(M, N)$.

DEFINITION 1.2. We say that $\varphi \in \mathscr{F}_{i}(M, p)$ is a $p$-invariant convex isometric folding if and only if $\varphi(M)$ can be embedded as a convex set in $\mathbb{R}^{m}$.

We denote the set of $p$-invariant convex isometric foldings of $M$ by $C_{i}(M, p)$. If $C_{i}(M, p) \neq \emptyset$, then for any covering map, $p: M \rightarrow N, C_{i}(M, p)$ is a subsemigroup of $C(M)$.

To solve our main problem we need the following:

(1) Robertson and Elkholy [5] proved that if $N$ is an $n$-smooth Riemannian manifold, $p: M \rightarrow N$ is its universal covering, and $G$ is the group of covering transformations of $p$, then $\mathscr{E}(N)$ is isomorphic as a semigroup to $\mathscr{F}_{i}(M, p) / G$.

(2) Elkholy [1] proved that if $N$ is an $n$-smooth Riemannian manifold, $p: M \rightarrow N$ is its universal covering, and $\varphi \in \mathscr{g}(N)$ such that $\varphi_{*}: \pi_{1}(N) \rightarrow \pi_{1}(N)$ is trivial, then the 
corresponding folding $\psi \in \mathscr{F}_{i}(M, p)$ maps each fiber of $p$ to a single point.

(3) Elkholy and Al-Ahmady [3] proved that under the same conditions of (2), if $N$ is a compact 2-manifold, then

$$
\frac{\operatorname{Vol} N}{\operatorname{Vol} \varphi(N)}=\frac{\operatorname{Vol} F}{\operatorname{Vol} \psi(F)}
$$

where $F$ is a fundamental region of $G$ in $M$.

2. Convex isometric folding and covering spaces. The next theorem establishes the relation between the set of convex isometric folding of a manifold, $C(N)$, and the set of $p$-invariant convex isometric folding of its universal covering space, $C_{i}(M, p)$.

THEOREM 2.1. Let $N$ be a manifold and $p: M \rightarrow N$ its universal covering. Let $G$ be the group of covering transformations of $p$. If $C(N) \neq \emptyset$, then $C(N)$ is isometric as a semigroup to $C_{i}(M, p) / G$.

Proof. Let $C(N) \neq \varphi$. Then by using (1), there exists an isomorphism $f$ from $\mathscr{F}_{i}(M, p) / G$ into $\mathscr{f}(N)$. Since $C_{i}(M, p)$ is a subsemigroup of $\mathscr{F}_{i}(M, p), C_{i}(M, p) / G$ is a subsemigroup of $\mathscr{F}_{i}(M, p) / G$.

Let $h=f \mid\left(C_{i}(M, P) / G\right)$. Since $C_{i}(M, p) / G$ is a semigroup, $h$ is a homeomorphism and also it is one-one. To show that $h$ is an onto map, we suppose that $\varphi \in C(N)$. Hence, $\varphi \in \mathscr{F}(N)$ and, consequently, there exists $\psi \in \mathscr{F}_{i}(M, p) / G$. Since $\varphi \in C(N)$, $\varphi_{*}$ is trivial and hence for all $x \in M, \psi(G, x)=\psi(x)$, and therefore $\psi \in C_{i}(M, p) / G$.

THEOREM 2.2. Let $N$ be a compact orientable 2-manifold and consider the universal covering space $\left(\mathbb{R}^{2}, P\right)$ of $N$. Let $\varphi \in C(N)$ and $\psi \in C_{i}\left(\mathbb{R}^{2}, p\right)$. Then for all $x, y \in \mathbb{R}^{2}$, $d(\psi(x), \psi(y)) \leq \Delta$, where $\Delta$ is the radius of a fundamental region for the covering space.

Proof. Elkholy [1] proved the truth of the theorem for $N=S^{2}$. So, we have to prove it for the connected sum of $n$-tori. First, let $N=T$ be a torus homomorphic to the quotient space obtained by identifying opposite sides of a square of length " $a$ " as shown in Figure 1(a)

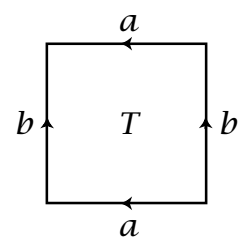

(a)

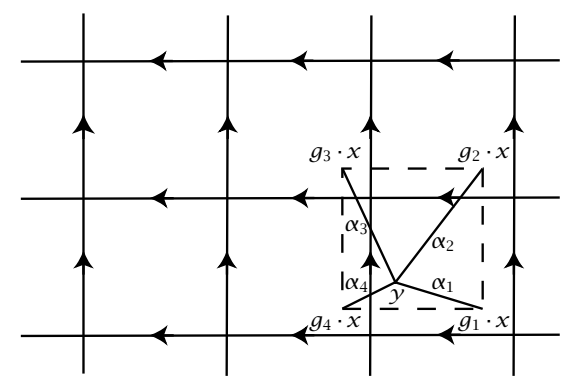

(b)

FIGURE 1. 
Suppose that $\varphi: T \rightarrow T$ is a convex isometric folding. Then $\varphi_{*}\left(\pi_{1}(T)\right)$ is trivial. By Theorem 2.1, there exists a convex isometric folding $\psi: \mathbb{R}^{2} \rightarrow \mathbb{R}^{2}$ such that for all $x$, $y \in \mathbb{R}^{2}$ and for all $g \in G, p(\psi(x))=p(\psi(g, x))$. Equivalently, for all $(P, Q) \in \mathbb{R}^{2}$ and for all $g \in \mathbb{Z} \times \mathbb{Z}$, there exists a unique $h \in \mathbb{Z} \times \mathbb{Z}$ such that $h \circ \psi(P, Q)=\psi(g(P, Q))$, i.e.,

$$
\psi(P, Q)+\left(\sqrt{2} \Delta m, \sqrt{2} \Delta n^{\prime}\right)=\psi(P+\sqrt{2} \Delta m, Q+\sqrt{2} \Delta n), \quad \text { where } m, n, m^{\prime}, n^{\prime} \in \mathbb{Z} .
$$

Consider any fundamental region $F$ of the covering space $\left(\mathbb{R}^{2}, p\right)$ of $T$, i.e., a closed square of length " $a$ " with sides identified as shown in Figure 1(b). Since $\varphi_{*}$ is trivial, by (2), for all $x \in \mathbb{R}^{2}, \psi(G, x)=\psi(x)$. Now, let $x$ and $y$ be distinct points of $\mathbb{R}^{2}$ such that $x=g \cdot y$ for all $g \in G$ and let $d(x, y)=\alpha_{1}$. Then there exists a point $x^{*}=g \cdot x$ such that

$$
d\left(y, x^{*}\right)=\min \left(\alpha_{i}\right), \quad \alpha_{i}=d\left(y, g_{i}, x\right), \quad i=1, \ldots, 4
$$

Thus, there are always four equivalent points $g_{i} \cdot x, i=1, \ldots, 4$ which form the vertices of a square of length " $a$ " and such that $d\left(g_{i} \cdot x, y\right) \leq 2 \Delta$. From Figure $1(\mathrm{~b})$, it is clear that $\max d\left(x^{*}, y\right) \leq \Delta$ and since $\psi$ is an isometric folding, by Robertson [4], $d(\psi(x), \psi(y)) \leq d(x, y)$, i.e.,

$$
d(\psi(x), \psi(y))=d\left(\psi\left(g_{i} \cdot x\right), \psi(y)\right) \leq d\left(g_{i} \cdot x, y\right)=d(x, y) \leq \Delta,
$$

and this proves the theorem for $N=T$.

Now, consider the connected sum of two tori, obtained as a quotient space of an octagon with sides identified as shown in Figure 2(a). The group of covering transformations $G$ is isometric to $\mathbb{Z} \times \mathbb{Z} \times \mathbb{Z} \times \mathbb{Z}$. Using the same previous technique, we can

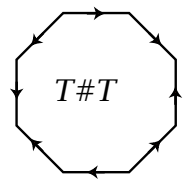

(a)

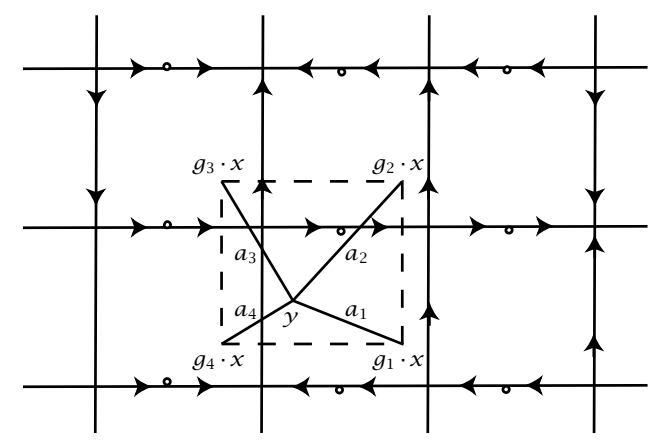

(b)

FIGURE 2.

obtain four equivalent points as the vertices of a square of diameter $2 \Delta$ such that $\max d\left(y, x^{*}\right) \leq \Delta$, and the result follows. This theorem, by using the above method, is true for the connected sum of $n$-tori. 
THEOREM 2.3. Let $N$ be a compact nonorientable 2-manifold and consider the universal covering space $(M, p)$ of $N$. Let $\phi \in C(N)$ and $\psi \in C_{i}(M, p)$. Then for all $x, y \in M, d(\psi(x), \psi(y)) \leq \Delta$, where $\Delta$ is the radius of a fundamental region for the covering space.

Proof. By Elkholy [2], the theorem is true for $N=p^{2}$ and $M=S^{2}$. Now, consider the connected sum of two projective planes, the Klein bottle $K$, homeomorphic to the quotient space obtained by identifying the opposite sides of a square as shown in Figure 3(a).

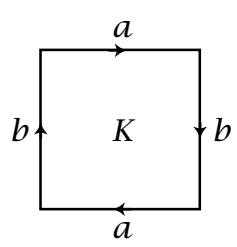

(a)

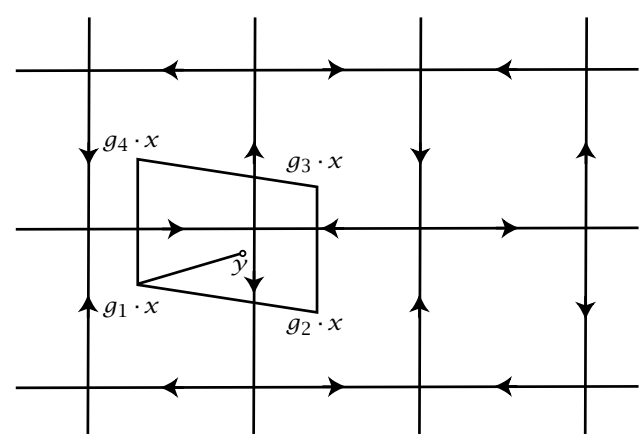

(b)

FIGURE 3.

Suppose that $\varphi: K \rightarrow K$ is a convex isometric folding. Then there exists a convex isometric folding $\psi: \mathbb{R}^{2} \rightarrow \mathbb{R}^{2}$ such that for all $x \in \mathbb{R}^{2}$ and $g \in G, p(\psi(x))=p(\psi(g$. $x)$ ). Equivalently, for all $(P, Q) \in \mathbb{R}^{2}$ and for all $g \in \mathbb{Z} \times \mathbb{Z}_{2}$, there exists a unique $h \in \mathbb{Z} \times \mathbb{Z}_{2}$ such that $h \circ \psi(P, Q)=\psi(g(P, Q))$, i.e.,

$$
\begin{aligned}
\psi(P, Q)+ & \left(\sqrt{2} \Delta m^{\prime}, \sqrt{2} \Delta n^{\prime}\right) \\
& =\psi\left(P+\sqrt{2} \Delta m, \sqrt{2} \Delta n+(-)^{m} Q\right), \text { where } m, n, m^{\prime}, n^{\prime} \in \mathbb{Z} .
\end{aligned}
$$

Any fundamental region $F$ of the covering space $\left(\mathbb{R}^{2}, p\right)$ of $K$ is a closed square of diameter $2 \Delta$ with the boundary identified as shown in Figure 3(b). Since $\varphi_{*}$ is trivial, for all $x \in R^{2}, \psi(G \cdot x)=\psi(x)$.

Now, let $x$ and $y$ be distinct points of $\mathbb{R}^{2}$ such that $y \neq g \cdot x$ for all $g \in G$, and let $d(x, y)=\alpha_{1}$. Thus, there exists a point $x^{*}=g \cdot x$ such that

$$
d\left(y, x^{*}\right)=\min \left(\alpha_{i}\right), \quad \alpha_{i}=d\left(y, g_{i} \cdot x\right), \quad i=1, \ldots, 4 .
$$

Thus, there are always four equivalent points $g_{i} \cdot x$ which form the vertices of a parallelogram such that the shortest diameter is of length less than $2 \Delta$.

Now, the point $y$ is either inside or on the boundary of a triangle of vertices $g_{1} \cdot x=$ $x, g_{2} \cdot x, g_{3} \cdot x$. Let $y^{\prime}$ be a point equidistant from the vertices of this triangle, i.e.,

$$
d\left(y^{\prime}, x\right)=d\left(y^{\prime}, g_{2} \cdot x\right)=d\left(y^{\prime}, g_{3} \cdot x\right)
$$


From Figure 3(b), it is clear that $d\left(y^{\prime}, x\right)<\Delta$ and, hence, $d\left(x^{*}, y\right)<\Delta$. Therefore,

$$
d(\psi(x), \psi(y))=d\left(\psi\left(g_{i} \cdot x\right), \psi(y)\right) \leq d\left(g \cdot x_{i}, y\right)=d\left(x^{*}, y\right)<\Delta
$$

and the result follows.

Now, let $N$ be the connected sum of three projective planes obtained as the quotient space of a hexagon with the sides identified in pairs as indicated in Figure 4(a). In this case, $\left(\mathbb{R}^{2}, p\right)$ is the universal cover of $N$ and $G \simeq \mathbb{Z} \times \mathbb{Z} \times \mathbb{Z}_{2}$. Using the same method as that used above, we can always have equivalent points $g_{i} \cdot x, i=1, \ldots, 4$ which form the vertices of a parallelogram whose shortest diameter is of length less than $2 \Delta$. From Figure 4(b), we can see that $\max d\left(y, x^{*}\right)<\Delta$ and the theorem is proved.

In general and by using the same technique, the theorem is also true for the connected sum of $n$-projective planes.

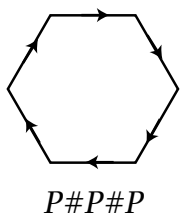

(a)

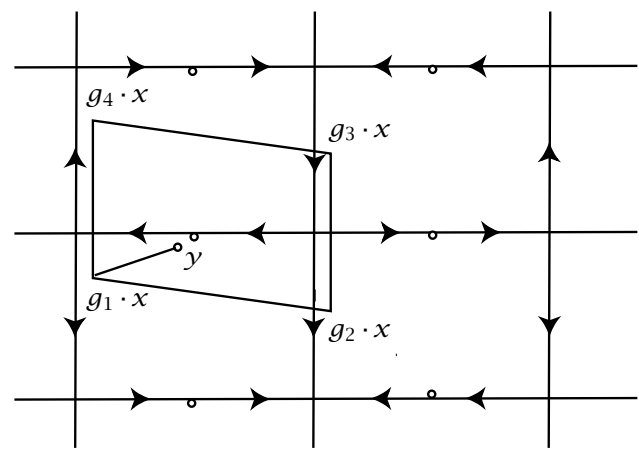

(b)

FIGURE 4.

3. Volume and convex folding. The following theorem succeeds in estimating the maximum volume we may have if we convexly folded a compact 2-manifold into itself.

THEOREM 3.1. The infimum of the ratio

$$
e_{N}=\frac{\operatorname{Vol} N}{\operatorname{Vol} \varphi(N)},
$$

where $N$ is a compact 2-manifold over all convex isometric foldings $\varphi \in C(N)$ of degree zero, is 4 .

Proof. Robertson [4] has shown that if $N$ is a compact 2-manifold, and $\varphi: N \rightarrow N$ is a convex isometric folding, any convex isometric folding is an isometric folding, then $\operatorname{deg} \varphi$ is \pm 1 or 0 . We consider only the case for which $\operatorname{deg} \varphi$ is zero otherwise $\varphi(N)$ cannot be embedded as a convex subset of $\mathbb{R}^{2}$ unless $N$ is. In this case, the set of singularities of $\varphi$ decomposes $N$ into an even number of strata, say $k$, each of which is homeomorphic to $\varphi(N)$ and, hence,

$$
\operatorname{Vol} N=k \operatorname{Vol} \varphi(N),
$$


that is, $e_{N}$ should be an even number. To calculate the exact value of $e_{N}$, consider first an orientable 2-compact manifold $N$. By using (1.1)

$$
e_{N}=\frac{\operatorname{Vol} F}{\operatorname{Vol} \varphi(F)}
$$

and this means that $e_{N}$ can be calculated by calculating the volume of $F$ and of its image $\varphi(F)$, but $F$ is a closed square of diameter $2 \Delta$ and $\varphi(F)$ is a closed subset of $F$ such that the distance $d\left(x, x^{\prime}\right)$ between any two points $x, x^{\prime} \in \varphi(F)$ is at most $\Delta$. The supremum of 2-dimensional volume of such set is $\phi(\Delta / 2)^{2}$ and, hence, $2<e_{N}$. But $e_{N}$ is an even number. Hence, $e_{N}=4$.

Now, let $N$ be a nonorientable 2-compact manifold, i.e., a connected sum of $n$ projective planes. Elkholy [2] proved the theorem for $n=1$.

The fundamental region in this case is a square or a rectangle of diameter $2 \Delta$ according to whether $n$ is even or odd. If $n$ is an even number, then

$$
\operatorname{Vol} F=2 \Delta^{2}
$$

and the result follows. Now, let $n$ be an odd number. Then $F$ is a rectangle of lengths $((n+1) / 2) a,((n-1) / 2) a$ and hence

$$
\operatorname{Vol} F=4 \Delta^{2} \sin \theta \cos \theta=4 \Delta^{2} \frac{a(n+1) / 2}{a \sqrt{\left(n^{2}+1\right) / 2}} \frac{a(n-1) / 2}{a \sqrt{\left(n^{2}+1\right) / 2}}=\frac{n^{2}-1}{n^{2}+1} 2 \Delta^{2} .
$$

Therefore, $e_{N}>2$ for all $n>1$. Since $e_{N}$ is an even number, $e_{N}=4$.

\section{REFERENCES}

[1] E. M. Elkholy, Isometric and topological folding of manifolds, Ph.D. thesis, Southampton University, England, 1982.

[2] _ Maximum isometric folding of the real projective plane, J. Natur. Sci. Math. 25 (1985), no. 1, 25-30. MR 87d:53081. Zbl 589.53052.

[3] E. M. Elkholy and A. E. Al-Ahmady, Maximum volume of isometric foldings of compact 2Riemannian manifolds, Proc. Conf. Oper. Res. and Math. Methods Bull. Fac. Sc. Alex. 26 A (1977), no. 1, 54-66.

[4] S. A. Robertson, Isometric folding of Riemannian manifolds, Proc. Roy. Soc. Edinburgh Sect. A 79 (1977), no. 3-4, 275-284. MR 58 7486. Zbl 418.53016.

[5] S. A. Robertson and E. M. Elkholy, Invariant and equivariant isometric folding, Delta J. Sci. 8 (1984), no. 2, 374-385.

ElKholy: Department of Mathematics, Faculty of Science, TANTA University, TANTA, EGYPT 


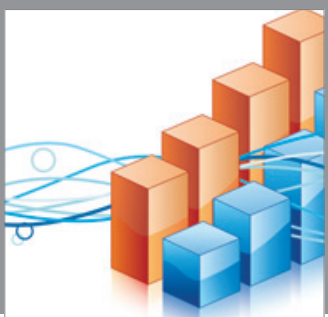

Advances in

Operations Research

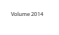

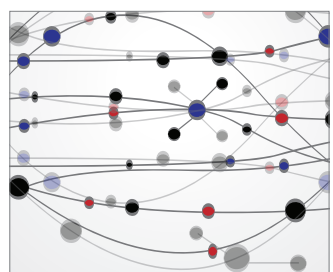

\section{The Scientific} World Journal
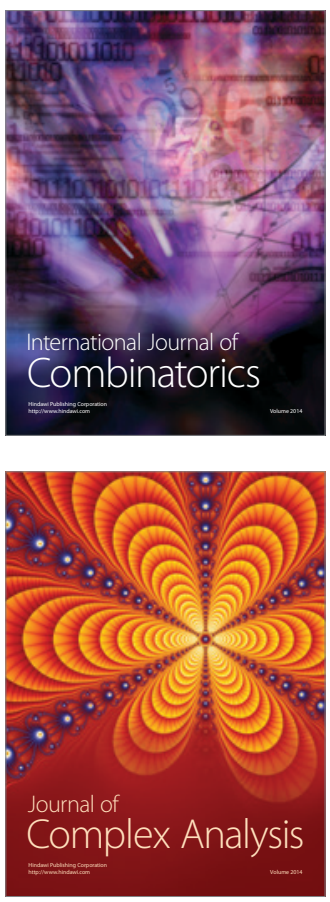

International Journal of

Mathematics and

Mathematical

Sciences
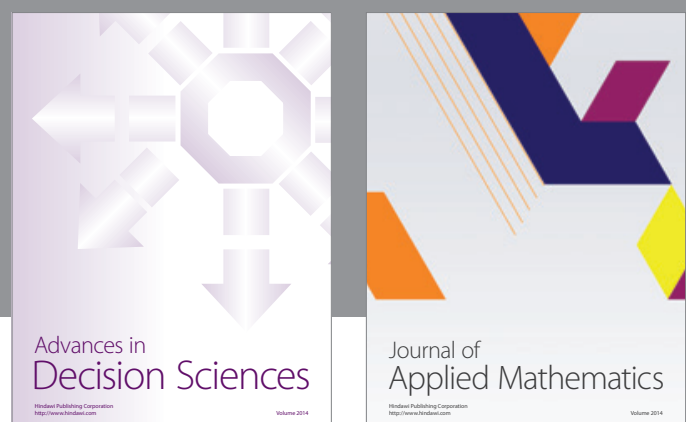

Journal of

Applied Mathematics
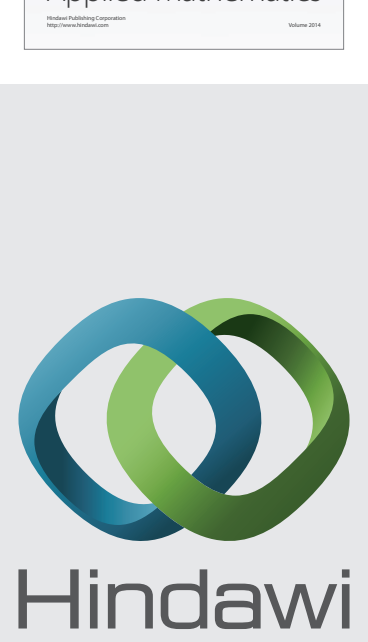

Submit your manuscripts at http://www.hindawi.com
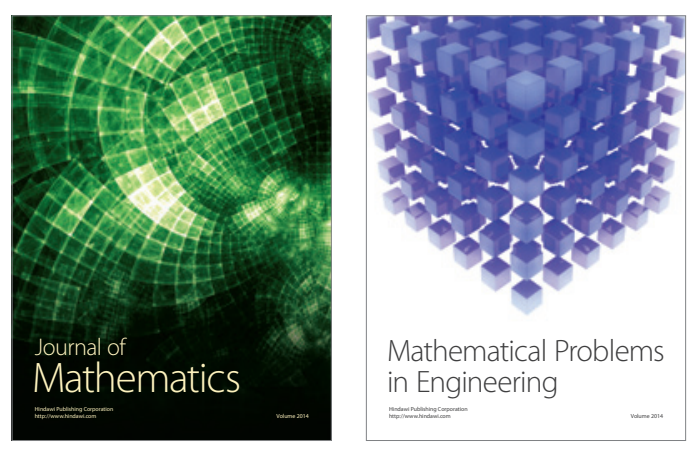

Mathematical Problems in Engineering
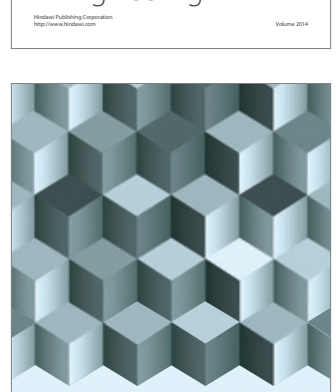

Journal of

Function Spaces
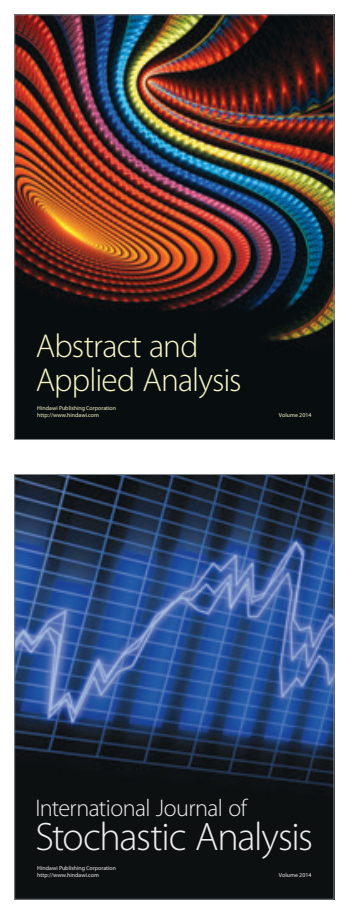

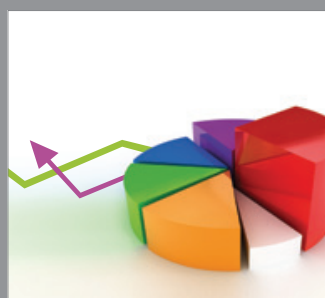

ournal of

Probability and Statistics

Promensencen
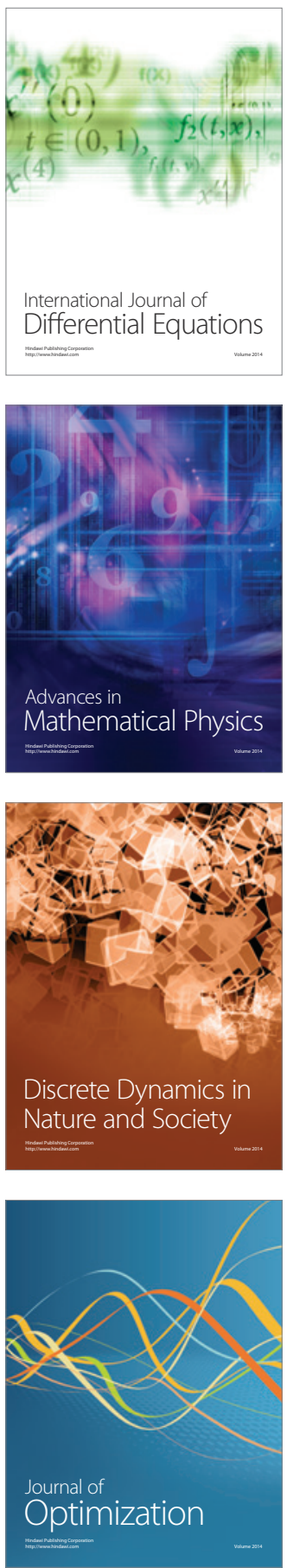